Research Paper

\title{
Pretreatment Nomograms for Local and Regional Recurrence after Radical Radiation Therapy for Primary Nasopharyngeal Carcinoma
}

\author{
Fo-ping Chen ${ }^{1 *}$, Li Lin ${ }^{*}$, Zhen-yu Qi ${ }^{1}$, Guan-qun Zhou1, Rui Guo1, Jiang Hu1 ${ }^{1}$ Ai-Hua Lin², Jun Ma ${ }^{\bowtie}$, Ying
} Sun ${ }^{1 \times}$

1. State Key Laboratory of Oncology in Southern China, Collaborative Innovation Center of Cancer Medicine, Department of Radiation Oncology, Cancer Center, Sun Yat-sen University, Guangzhou 510060, People's Republic of China;

2. Department of Medical Statistics and Epidemiology, School of Public Health, Sun Yat-sen University, Guangzhou, People's Republic of China.

* These authors contributed equally to this work.

$\triangle$ Corresponding authors: Dr. Ying Sun, Department of Radiation Oncology, Sun Yat-sen University Cancer Center, State Key Laboratory of Oncology in South China, Collaborative Innovation Center of Cancer Medicine, 651 Dongfeng Road East, Guangzhou 510060, People's Republic of China. Telephone: +86-020-87342253 Fax: +86-020-87343295 E-mail: sunying@sysucc.org.cn Jun Ma, Department of Radiation Oncology, Sun Yat-sen University Cancer Center, State Key Laboratory of Oncology in South China, Collaborative Innovation Center of Cancer Medicine, 651 Dongfeng Road East, Guangzhou 510060, People's Republic of China. Telephone: +86-20-87343469 Fax: +86-20-87343295 E-mail: majun2@mail.sysu.edu.cn

(C) Ivyspring International Publisher. This is an open access article distributed under the terms of the Creative Commons Attribution (CC BY-NC) license (https://creativecommons.org/licenses/by-nc/4.0/). See http://ivyspring.com/terms for full terms and conditions.

Received: 2017.03.25; Accepted: 2017.07.01; Published: 2017.08.03

\begin{abstract}
Background: The aim of this study was to build nomograms to predict local recurrence (LR) and regional recurrence (RR) in patients with nasopharyngeal carcinoma (NPC) underwent intensity-modulated radiation therapy (IMRT).

Patients and Methods: A total of 1811 patients with non-metastatic NPC treated with IMRT (with or without chemotherapy) between October 2009 and February 2012 at our center were involved for building the nomograms. Nomograms for LR-free rate and RR-free rate at 3- and 5year were generated as visualizations of Cox proportional hazards regression models, and validated using bootstrap resampling, estimating discrimination and calibration.

Results: With a median follow up of 49.50 months, the 3- and 5- year LR-free rate were 95.43\% and $94.30 \%$ respectively; the 3 - and 5- year RR-free rate were $95.94 \%$ and $95.41 \%$ respectively. The final predictive model for LR included age, the neutrophil/leukocyte ratio (NWR), pathological type, primary gross tumor volume, maxillary sinus invasion, ethmoidal sinus invasion and lacerated foramen invasion; the model for RR involved NWR, plasma Epstein-Barr virus (EBV) DNA copy number, cervical lymph node volume and $\mathrm{N}$ category. The models showed fairly good discriminatory ability with concordance indices (c-indices) of 0.76 and 0.74 for predicting LR and RR, respectively, as well as good calibration. The proposed stratification of risk groups based on the nomograms allowed significant distinction between Kaplan-Meier curves for LR and RR.

Conclusions: The proposed nomograms resulted in more-accurate prognostic prediction for LR and RR with a high concordance, hence to inform patients with high risk of recurrence on more aggressive therapy. The prognostic nomograms could better stratify patients into different risk groups.
\end{abstract}

Key words: Nomogram, Local Recurrence, Regional Recurrence, Nasopharyngeal Carcinoma, Intensity-Modulated Radiation Therapy. 


\section{Introduction}

Nasopharyngeal carcinoma (NPC) arises from the nasopharyngeal epithelium and has an extremely unbalanced geographical distribution with high incidence in some areas of southern China, northern Africa and Alaska [1]. Because of the anatomic constraints and its high radiosensitivity, radiotherapy is the primary and only curative treatment modality for non-metastatic NPC [2]. Although prognosis of patients with NPC has improved, loco-reginal recurrence (LRR) of disease is still a critical problem just second to distant metastasis. Unfortunately, it is not clear what the optimal interval and modalities for detecting LRR without symptoms are. Despite the various surveillance strategies to monitor recurrence for NPC after IMRT, however, it is not clear whether early detection of a recurrence would improve prognosis. Therefore, a prognostic tool enabling accurately predicting LRR are required for NPC.

Substantive research has indicated a variety of clinicopathological covariates such as $\mathrm{T} / \mathrm{N}$ category, plasma Epstein-Barr virus (EBV)-DNA copy number, the primary gross tumor volume and pretreatment LDH are associated with LRR [12-16]. However, none of them is sufficiently accurate in routine practice to identify patients at risk of recurrence. Therefore, it may be beneficial to integrate a variety of comprehensive clinicopathological information, including anatomic factors, host factors and pathological factors, in a manner that enables accurate prediction of LRR. Such tools may help to optimize individualized treatment and follow-up strategies to further increase loco-regional control in NPC.

Nomogram is a reliable, pragmatic predictive tool that provides a simple graphical representation of statistical predictive models to quantify individual risk by incorporating a variety of risk factors for a clinical event [22, 23]. Nomograms have been developed for many types of cancer and been proven to provide more precise predictions than the traditional TNM staging system and univariate prognostic factors [22, 23, 24]. To date, nomograms have not yet been devised to predict LR or RR for NPC receiving radical IMRT. In this study, we undertook a large-scale study, with the aim of generating reliable, pragmatic nomograms for LR-free and RR-free rate using comprehensive clinical and pathological variables.

\section{Materials and Methods}

\section{Patient characteristics}

A database with a total of 1811 consecutive newly biopsy-proven, non-metastatic NPC treated with IMRT at our center between October 2009 and
February 2012 was prospective maintained. The medical data including electronic medical records and imaging data of each patient was firstly retrospectively collected, and then routinely prospectively followed up on the basis of internal schedule. Moreover, telephone follow-up was performed for who did not appear to follow-up at our center or when we think it was necessary for other reasons. Routine staging workup including a complete medical history, physical examination, hematology and biochemistry profiles, nasopharyngeal fiberoptic endoscopy, nasopharyngeal and neck MRI, chest radiography, abdominal sonography and whole body bone scan using 99mTc-methyldiphosphonate single photon emission computed tomography (SPECT) were performed for all patients. All patients were restaged according to the 7th edition of the International Union against Cancer/American Joint Committee on Cancer (UICC/AJCC) staging system [34]. This study was approved by the institutional ethics committee and all the methods were carried out in accordance with The Code of Ethics of the World Medical Association. Informed consent was obtained from every patient prior to therapy.

\section{Treatment}

The nasopharyngeal and neck tumor target volumes of all patients were treated using radical IMRT in the process of the entire treatment course. Target volumes were delineated slice-by-slice on the treatment planning CT scans using an individualized delineation protocol in accordance with International Commission on Radiation Units and Measurements reports 62 and 83. Prescribed doses were 66-72Gy at 2.12- $2.43 \mathrm{~Gy} /$ fraction to planning target volume (PTV) of primary gross tumour volume (GTVp), 64-70 Gy to PTV of GTV of involved lymph nodes (GTVnd), 60-63 Gy to PTV of high-risk clinical target volume (CTV1), and 54-56 Gy to PTV of low-risk clinical target volume (CTV2). All targets were treated simultaneously using the simultaneous integrated boost technique; other details of the techniques used at our center have previously been reported [25].

According to institutional guidelines, we recommended RT alone for stage I, concurrent chemoradiotherapy (CCRT) for stage II, and CCRT \pm neoadjuvant/adjuvant chemotherapy for stage III to IVA-B. Of the 1811 patients, $256(14.1 \%)$ received IMRT alone; 1356 (74.9\%) received concurrent chemotherapy, of whom $675(37.3 \%)$ received neoadjuvant chemotherapy, $42(2.3 \%)$ received adjuvant chemotherapy; and 199 (11.0\%) received neoadjuvant chemotherapy +/- adjuvant chemotherapy. Neoadjuvant/adjuvant chemotherapy 
consisted of cisplatin with 5-fluorouracil, cisplatin with docetaxel, or cisplatin with 5-fluorouracil and docetaxel were administered at intervals of 3 weeks for two to four cycles. Concurrent chemotherapy was the single drug cisplatin administered every three weeks or weekly during the treatment course of radiotherapy.

\section{Patient follow-up}

Each patient was assessed for treatment response and toxicity every week during treatment, every 3 months during the first 2 years after radiotherapy and every 6 months thereafter (or until death). The main endpoints of interest were local recurrence (LR) and regional recurrence (RR), defined as tumor residual or relapse at least 3 months after initial IMRT. All LR were confirmed via fiber-optic endoscopy and biopsy or MRI scan (or both) of nasopharynx and skull base showing progressive bone erosion and soft tissue swelling. All RR were diagnosed by clinical examination of neck and by fine-needle aspiration, or MRI of neck (or both). Interval times for LR and RR were calculated and defined as the duration between commencement of radiotherapy and first detection of any evidence of LR and RR.

\section{Designing of predictive nomograms}

Univariate analysis was performed using Cox proportional hazard regression models to determine the hazard ratios for LR and RR. The complexity of the models was controlled using the Akaike information criteria. Nomograms for the probabilities of LR and RR at 36 and 60 months were generated. Values for each of the model covariates were mapped to points on a scale axis ranging from $0-100$. The total points summed for each model covariate were mapped to the probability of LR or RR associated with the specific combination of covariate values [22, 23]. The predictive accuracy of the nomograms was evaluated by assessing discrimination and calibration, and measures of predictive accuracy were validated using a 1000 bootstrap resampling method. Discrimination between patients with or without LR and RR was assessed using the concordance index (c-index), a variable equivalent to the area under the receiver operating characteristic curve (AUC). A c-index of 0.5 indicates the random chance of correctly predicting the outcome; 1.0 indicates perfect distinction between predicted and actual outcomes. Biases of predictive performance were first estimated using bootstrap samples, then corrected with apparent measures to produce bias-corrected values to avoid overfitting [24]. Calibration was performed using a calibration curve incorporating the model-predicted and actual probabilities.

Finally, we determined the cutoff values of nomogram generated scores with which patients were evenly stratified into three or four risk groups: low, middle, high risk and very high. The predictive value of the classifications were estimated by means of the hazard ratio (HR) and its 95\% confidence interval (95\% CI) between the groups thereby generated and LR and RR.

\section{Statistical analysis}

Time-to-event data were described using Kaplan-Meier curves and the differences were compared with the log-rank test. Receiver operating characteristic (ROC) curve analyses were applied to determine optimal cut-off points, which were defined by maximizing the conditional Youden score, for continuous variables with respect to loco-regional recurrence. The multivariate Cox proportional hazards model was used to estimate hazard ratios (HRs) and 95\% confidence intervals (CIs). Univariate and Multivariate Cox proportional hazard regression models were conducted using SPSS software, version 22.0 (SPSS, Chicago, IL, USA). Establishment and verification of nomograms were implemented using the open source software R-version 3.2.5 with rms packages (Design, Vienna, Austria). All tests were two-sided; $p$-values $<0.05$ were considered statistically significant.

\section{Results}

\section{Loco-regional recurrence and survival}

With a median follow up of 49.50 months (ranging, 1.3-76.4 months), 90 patients developed LR and 75 patients developed regional recurrence $R R$, respectively. The 5- year LR-free rate and RR-free rate were $94.30 \%$ and $95.41 \%$ respectively, with median durations of 22.99 and 20.20 months to LR and RR respectively.

The detailed clinicopathological characteristics and treatment factors for the 1811 patients are listed in Table 1. The male: female ratio was 3:1 (1351 males, 460 females); patients ranged in age from 14 to 78 years-old (median, 45 years). Histological examination revealed $94.4 \%$ of patients had undifferentiated NPC, and 5.6\% had differentiated NPC. The T category distribution was T1: 326 (18\%); T2: 282 (15.6\%); T3: 864 (47.7\%); and T4: 339 (18.7\%). Overall, $308(17 \%)$ patients staged N0; $1062(58.6 \%)$ staged N1; $276(15.2 \%)$ staged N2; $165(9.1 \%)$ staged N3. 
Table 1. Patient characteristics and treatment factors $(n=1811)$

\begin{tabular}{|c|c|c|}
\hline Characteristic & No. $(n=1811)$ & $\%$ \\
\hline \multicolumn{3}{|l|}{ Patient and tumor characteristics } \\
\hline \multicolumn{3}{|l|}{ Sex } \\
\hline Male & 1351 & 74.6 \\
\hline Female & 460 & 25.4 \\
\hline \multicolumn{3}{|l|}{ Age (years) } \\
\hline Median & 45 & \\
\hline Range & $14-78$ & \\
\hline \multicolumn{3}{|l|}{ Smoking } \\
\hline Yes & 659 & 63.6 \\
\hline No & 1152 & 36.4 \\
\hline \multicolumn{3}{|l|}{ WHO histologic type } \\
\hline Differentiated & 101 & 5.6 \\
\hline Undifferentiated & 1710 & 94.4 \\
\hline \multicolumn{3}{|l|}{$\mathrm{T}$ category } \\
\hline $\mathrm{T} 1$ & 326 & 18 \\
\hline $\mathrm{T} 2$ & 282 & 15.6 \\
\hline $\mathrm{T} 3$ & 864 & 47.7 \\
\hline $\mathrm{T} 4$ & 339 & 18.7 \\
\hline \multicolumn{3}{|l|}{$\mathrm{N}$ category } \\
\hline No & 308 & 17 \\
\hline N1 & 1062 & 58.6 \\
\hline N2 & 276 & 15.2 \\
\hline N3 & 165 & 9.1 \\
\hline \multicolumn{3}{|l|}{ Stage } \\
\hline I & 99 & 5.5 \\
\hline II & 381 & 21 \\
\hline III & 856 & 47.3 \\
\hline IV & 475 & 26.2 \\
\hline \multicolumn{3}{|l|}{ Treatment factors } \\
\hline IMRT prescribed dose (Gy/Fr) & $66-72 / 28-33$ & \\
\hline \multicolumn{3}{|l|}{ Chemotherapy (no/yes) } \\
\hline None & 256 & 14.1 \\
\hline $\mathrm{CCRT}+/-\mathrm{ACT}$ & 681 & 37.6 \\
\hline $\mathrm{CCRT}+\mathrm{NCT}$ & 675 & 37.3 \\
\hline $\mathrm{NCT}+/-\mathrm{ACT}$ & 199 & 11.0 \\
\hline
\end{tabular}

\section{Predictive nomogram for LR}

The results of univariate and multivariate analyses for LR are summarized in Table 2 . Twenty-three factors were significantly associated with LR in univariate analysis $(P<0.05)$. However, only six factors remained significant in multivariate analyses. The influential predictors identified in the Cox proportional hazards regression models in addition to volume of GTVp were included to generate the nomogram for LR. As shown in Figure 1A, the factors finally included in the model for LR were: age, the neutrophil/leukocyte ratio (NWR), pathological type, primary gross tumor volume, maxillary sinus invasion, ethmoidal sinus invasion and lacerated foramen invasion.

The c-index of the nomogram for LR was 0.76 (95\% CI; 0.71 to 0.81 ), which was significantly better than that of the TNM classification $(0.61 ; 95 \% \mathrm{CI}, 0.55$ to $0.67 ; P<0.001)$. The predicted probabilities at $3-$ and 5 - years obtained from the bootstrap correction and actual probabilities of LR are illustrated in the calibration plot (Figure 2A). Both plots showed good agreement between predicted and observed LR, with the calibration curves close to the 45 degree broken line indicating the nomogram was calibrated accurately.

Table 2. Predictive factors for $L R$ in univariate and multivariate analysis

\begin{tabular}{|c|c|c|c|c|}
\hline & Univariate & Mult & ariate analy & \\
\hline & $\begin{array}{l}\text { analysis } \\
(P)\end{array}$ & $\overline{\mathrm{HR}}$ & $95 \% \mathrm{CI}$ & $P$ \\
\hline Sex (Male/Female) & 0.087 & & & NS \\
\hline Age ( $>46 / \leq 46$ years) & 0.081 & 1.54 & $1.00-2.35$ & 0.049 \\
\hline Smoking (No/Yes) & 0.901 & & & NS \\
\hline $\mathrm{WBC} / \times 10^{9}(>7.72 / \leq 7.72)$ & 0.014 & & & NS \\
\hline Neu $/ \times 10^{9}(>4.53 / \leq 4.53)$ & 0.005 & & & NS \\
\hline $\operatorname{Lym} / \times 10^{9}(>1.50 / \leq 1.50)$ & 0.064 & & & NS \\
\hline NWR $(>0.64 / \leq 0.64)$ & $<0.001$ & 1.86 & $1.20-2.87$ & 0.005 \\
\hline $\operatorname{NLR}(>2.36 / \leq 2.36)$ & $<0.001$ & & & NS \\
\hline $\mathrm{LDH} / \mathrm{U} / \mathrm{L}(>187 / \leq 187)$ & 0.052 & & & NS \\
\hline $\mathrm{CRP} / \mathrm{mg} / \mathrm{L}(>2.36 / \leq 2.36)$ & 0.076 & & & NS \\
\hline $\begin{array}{l}\text { EBV-DNA /copies/ml (> } \\
2830 / \leq 2830)\end{array}$ & 0.014 & & & NS \\
\hline $\begin{array}{l}\text { Pathological type } \\
\text { (differentiated/undifferentiated) }\end{array}$ & 0.015 & 0.50 & $0.26-0.96$ & 0.038 \\
\hline Nasopharynx & 0.024 & & & NS \\
\hline Nasal cavity & 0.096 & & & NS \\
\hline Levator veli palatine muscle & 0.034 & & & NS \\
\hline Tensor veli palatini muscle & 0.045 & & & NS \\
\hline Vertebral anterior muscle & 0.019 & & & NS \\
\hline Parapharyngeal space & 0.016 & & & NS \\
\hline Infratemporal fossa & 0.599 & & & NS \\
\hline Pterygoid process & $<0.001$ & & & NS \\
\hline Base of sphenoid bone & 0.107 & & & NS \\
\hline Maxillary sinus & $<0.001$ & 3.33 & $1.42-7.81$ & 0.006 \\
\hline Sphenoid sinus & $<0.001$ & & & NS \\
\hline Ethmoidal sinus & $<0.001$ & 2.98 & $1.39-6.40$ & 0.005 \\
\hline Foramen Lacerated & $<0.001$ & 2.40 & $1.48-3.88$ & $<0.001$ \\
\hline Foramen Ovale & 0.001 & & & NS \\
\hline Cavernous sinus & $<0.001$ & & & NS \\
\hline Medial Pterygoid & 0.014 & & & NS \\
\hline External Pterygoid & 0.226 & & & NS \\
\hline Clivus & $<0.001$ & & & NS \\
\hline Petrous Apex & 0.001 & & & NS \\
\hline Great Wing of Sphenoid Bone & $<0.001$ & & & NS \\
\hline $\begin{array}{l}\text { Primary gross tumor volume / cc } \\
\leq 21.33\end{array}$ & Ref. & Ref. & & \\
\hline $21.33-75.50$ & 0.058 & & & NS \\
\hline$>75.50$ & $<0.001$ & & & NS \\
\hline $\begin{array}{l}\text { Cumulative dose to GTVp /Gy } \\
(>68 / \leq 68)\end{array}$ & 0.278 & & & NS \\
\hline Chemotherapy & & & & \\
\hline None & Ref. & Ref. & & \\
\hline Neoadjuvant \pm Concurrent & 0.105 & & & NS \\
\hline Concurrent & 0.108 & & & NS \\
\hline
\end{tabular}

\section{Predictive nomogram for $\mathbf{R R}$}

The univariate and multivariate analyses for RR are summarized in Table 3 . Univariate analyses indicated that $\mathrm{C}$-reactive protein (CRP), plasma 
EBV-DNA copy number, $\mathrm{N}$ category and volume of GTVnd were significant for RR. CRP had no significant value in multivariate analyses, while NWR become significant in the multivariate analysis when combined with other factors and adjusted using the stepwise regression method. NWR, EBV-DNA copy number, cervical lymph node volume and $\mathrm{N}$ category were finally included to generate the nomogram for RR (Figure 1B).

The c-index of the predictive model for RR was 0.74 (95\% CI; 0.68 to 0.80 ), which was significantly higher than that of TNM classification $(0.64 ; 95 \% \mathrm{CI}$, 0.58 to $0.70 ; P<0.001)$. The calibration curves for the probability of RR at 3- and 5-years corresponded to the 45 degree broken line (Figure 2B), indicating the nomogram provided perfect prediction of RR.

\section{Stratifying risk for individual patients using nomograms}

All patients were stratified into different risk groups (4 groups for LR; 3 groups for RR) according to the total points summed for each model covariate. The cutoff values of the nomogram-generated scores for stratification of LR were 142, 221, and 304; and the scores for stratification of RR were 93 and 219. These stratifications could effectively discriminate LR and $\mathrm{RR}$ for the proposed risk groups (Figure 3). The log-rank test reveals that the survival functions factored by prognostic categories are significantly different $(P<0.0001)$. It is worth noting that high-middle-risk patients had increased risk of loco-regional failure, with $\mathrm{HR}$ of $2.07(95 \% \mathrm{CI}$, 1.71-2.50) for $\mathrm{LR}$, and 3.11 (95\% CI, 1.98-4.89) for RR.

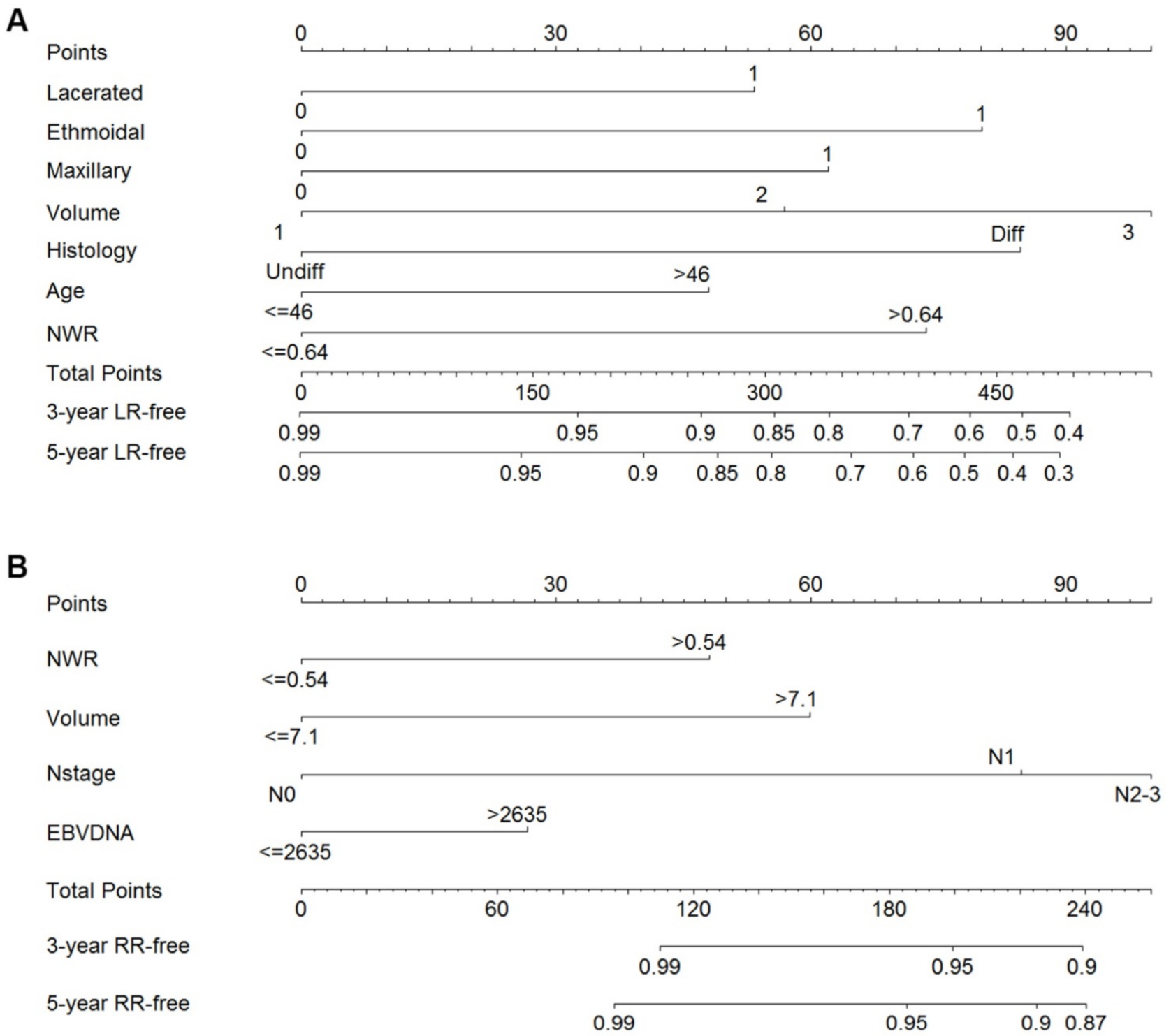

Figure 1. Nomograms of non-metastatic NPC patients after radical radiotherapy for LR-free survival (A) and RR-free survival (B). Undiff: Undifferentiated; Diff: Differentiated; NWR, neutrophil/leukocyte ratio; LDH, Lactate dehydrogenase; EBV, Epstein-Barr virus; LR, local recurrence; RR, regional recurrence. 
A



Nomogram-predicted LR-free probability

B

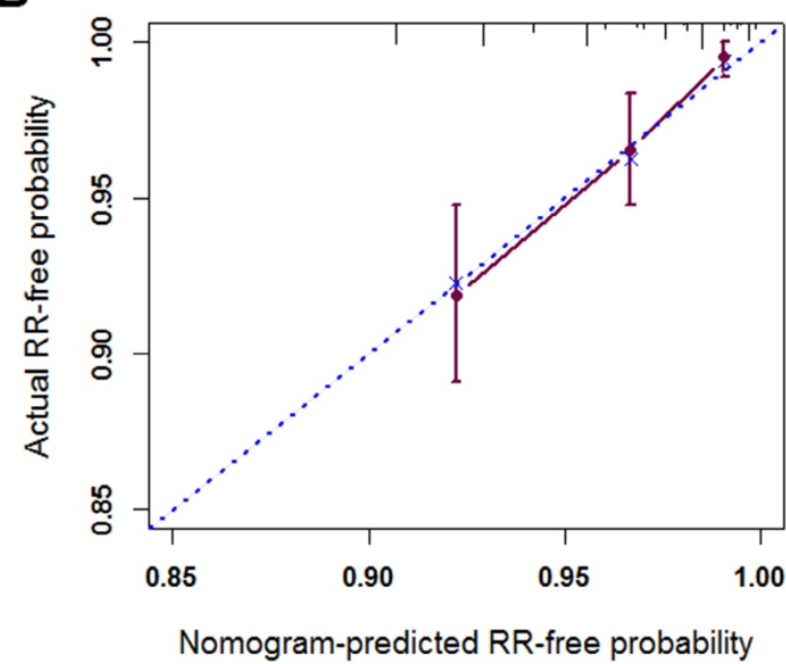

5-year calibration curve

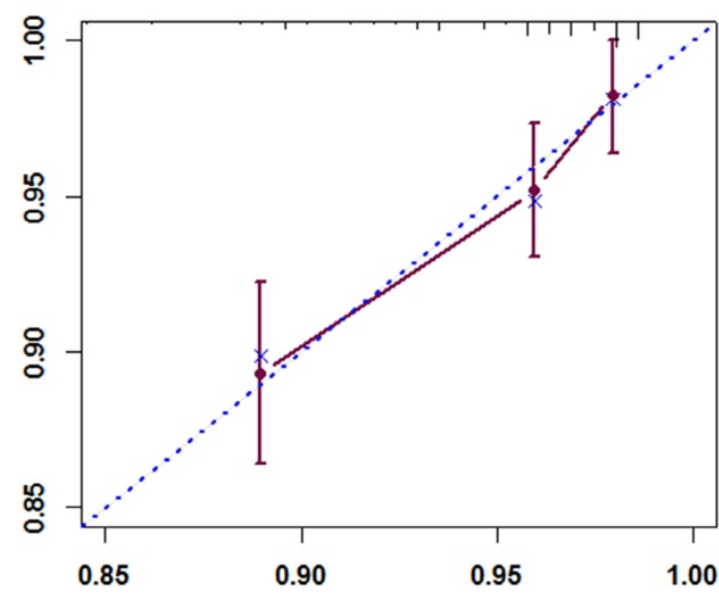

Nomogram-predicted LR-free probability

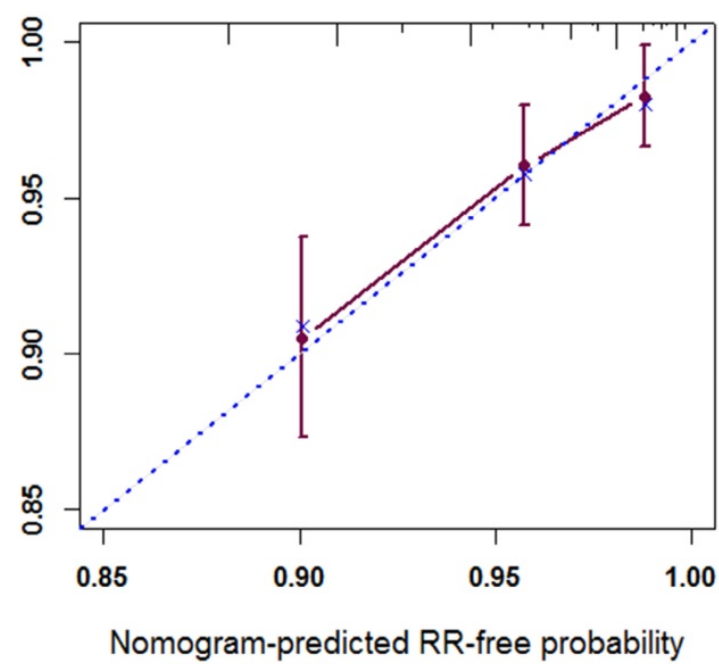

Figure 2. The calibration curves for predicting LR-free survival (A) and RR-free survival (B) at 3 years and 5 years. Nomogram-predicted probability of recurrence is plotted on the $x$-axis; actual recurrence-free probability is plotted on the $y$-axis. $L R$, local recurrence; $R R$, regional recurrence.

A LR stratified by nomogram

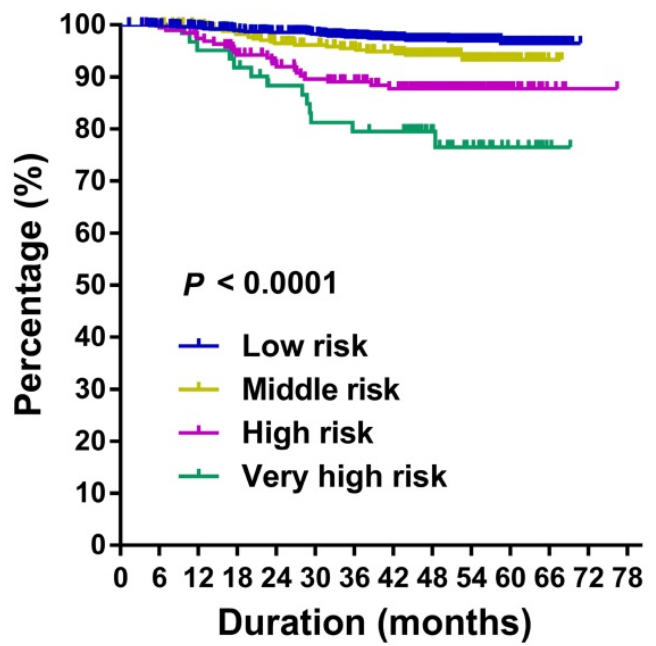

B

RR stratified by nomogram

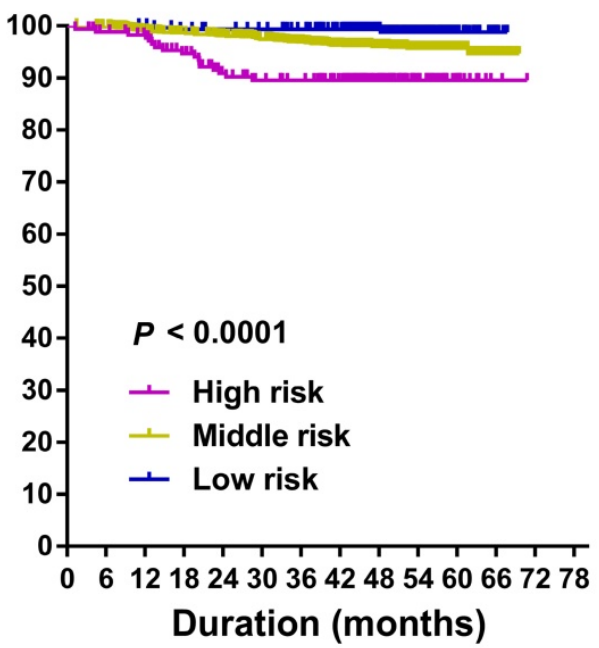

Figure 3. Kaplan-Meier curves of risk group stratification for LR (A) and RR (B). LR, local recurrence; RR, regional recurrence. 
Table 3. Predictive factors for $R R$ in univariate and multivariate analysis

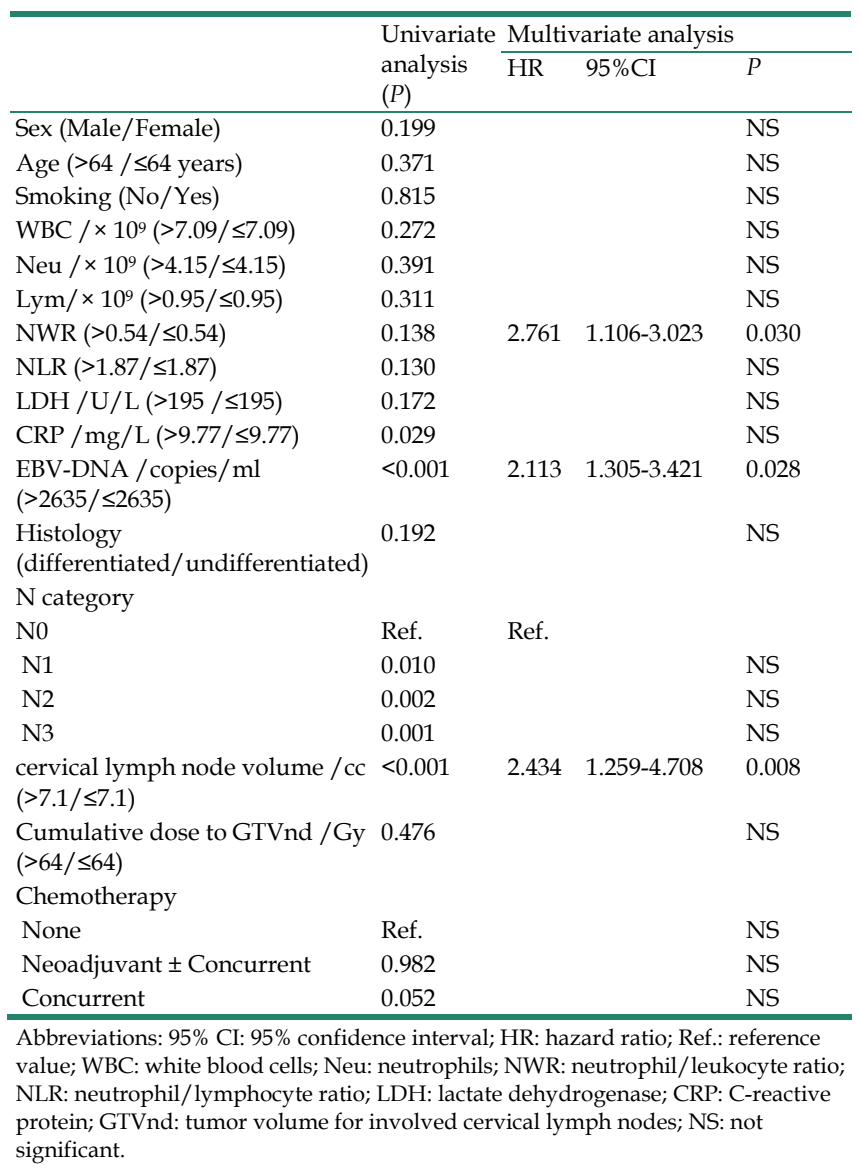

\section{Discussion}

In this study, we generated nomograms using widely-assessed clinicopathological information to predict the probability of LR-free and RR-free survival for patients with NPC after radical radiation therapy. Both models were well generated: the nomograms for LR and RR had c-indexes of 0.76 and 0.74, respectively, indicating the discriminative performance of the models was close to perfect. The calibration curves for the models intuitively suggested good agreement between the predictions and observations, supporting the goodness-of-fit of the nomogram predictions to the data. To our knowledge, these are the first nomograms developed to predict the probability of LR-free and RR-free rate for patients with NPC after radical IMRT.

The TNM staging system, in which tumor size, tumor invasion and the extent of lymph node involvement are stratified, is the most commonly used tool for clinicians to predict the prognosis of patients with NPC. However, with the widespread application of IMRT, the loco-regional control rate for NPC has greatly improved as IMRT offers excellent target volume coverage and sparing for the normal tissues adjacent to the targets [17], which may reduce the predictive value of $T$ category for $L R$ and $R R$ to a certain extent [20]. Furthermore, varieties of clinicopathological covariates - in addition to TNM stage - are associated with LR and RR [12-16]. On the other hand, the nomograms for LR-free and RR-free rate generated in this study include all relevant covariates and enable accurate prediction of LR and RR. The c-indexes of the nomograms were significantly higher than those of the TNM classification, indicating the significantly better predictive capacity of the nomograms.

The risk factors identified for LR were age, NWR, pathological type, primary gross tumor volume, maxillary sinus invasion, ethmoidal sinus invasion and lacerated foramen invasion. NWR is one of the major human inflammatory indicators and serve as key effectors in the first-line of host defense against infectious microorganisms. Substantial evidence indicates inflammation is a critical component that promotes tumor progression [30, 31]. High NWR is also an independent prognostic factor in NPC [31]. Neutrophils in the peripheral circulation and tumor microenvironment can produce proangiogenic factors, such as vascular endothelial growth factor, that stimulate tumor development [31]. Some cytokines involved in cancer-related inflammation and myeloid growth factors produced by cancer cells can induce neutrophilia [31].

The primary gross tumor volume has also been proven to be relevant to $\operatorname{LR}[4,13,14]$. Many patients with a large primary gross tumor volume experience tumor hypoxia, which is strongly associated with disease progression and resistance to therapy $[28,29]$. Besides, a larger primary gross tumor volume often reflects a heavier tumor cell burden, thus may further contribute to radioresistance. Furthermore, a larger primary gross tumor volume indicates the tumor lies closer adjacent to the organs at risk (OARs), and dose reductions may be necessary to protect critical normal tissues. Therefore, all of these adverse factors may contribute to increased risk of LR.

Certain sites invasion including maxillary sinus, ethmoidal sinus and lacerated foramen were found to be adverse factors for local recurrence in our series. These structures have close relation with the surrounding anatomic structures, and can be involved by tumor through mucosa crawl or direct invasion. IMRT can create a more conformal distribution around the targets than two-dimensional (2-D) radiation therapy, and the high dose region is defined by the precise three-dimensional (3-D) sectional anatomy. Thus, result in smaller CTV with IMRT. Therefore, it has higher chance to local recurrence for 
diseases with these structures involved.

The variables identified for the nomogram for RR-free rate were NWR, EBV-DNA copy number, cervical lymph node volume and $\mathrm{N}$ category. A recent Medline review by Wang et al. [32] stressed the important role of tumor-associated neutrophils (TANs) in the development and progression of cancer. TANs can promote tumor metastasis (including regional and distant metastasis) via various mechanisms. For example, matrix metalloproteinase 9 (MMP-9) is a type IV collagen that can be secreted by tumor cells and TANs in the tumor microenvironment. MMP-9 is involved in a wide range of physiological and pathological process in the body, such as wound healing, angiogenesis and inflammatory responses. Abnormal expression of MMP-9 is closely associated with metastasis in many types of cancer [32]. However, while it is not yet known whether MMP-9 produced by tumor cells promotes tumor angiogenesis and metastasis, MMP-9 produced by inflammatory cells clearly promotes angiogenesis and tumor progression [32].

The relevance of plasma EBV-DNA copy number to RR has been reported in several studies [12]. Lin et al. [12] demonstrated the presence of EBNA-1 DNA in peripheral blood cells was predictive of a high risk of metastasis, and the circulating EBV DNA copy number correlates with tumor stage and the likelihood of recurrence [12]. They defined a pretreatment cut-off value of 1500 copies per milliliter, and concluded that plasma EBV DNA represents a useful molecular marker for prediction of recurrence in NPC [12].

The cervical lymph node volume was significant for RR in univariate and multivariate analysis. Patients with larger cervical lymph nodes have higher incidence of cervical nodal necrosis and should receive a higher radiation dose. Besides, although all patients are immobilized during radiation using a thermoplastic head and shoulder mask, the neck is the least fixed region of the irradiation area. Therefore, large baseline shifts may occur during the long-time treatment course, which may result in under-dosing of the cervical lymph node.

Moreover, the risk group stratifications generated using the nomogram-generated scores could effectively discriminate for LR and RR. Aggressive therapy to patients with high risk of recurrence according to our nomograms may be proposed. Neoadjuvant chemotherapy before radiotherapy could reduce tumor volume, and improve gross tumor volume coverage and tumor control. Adaptive radiotherapy (ART) adjusts the treatment plan to systematic changes observed during $\mathrm{RT}$, and restores the target dose in the case of anatomic changes, which may also improve tumor control for the high-risk group.

The nomograms established in this study possess a number of limitations. The nomograms were not subjected to external validation, as we did not acquire a validation cohort. However, internal validation was applied using bootstrap resampling to avoid over-fitting of the data. Future validation suing external prospective data of larger cohorts are warranted to confirm these results.

\section{Conclusion}

In conclusion, the nomograms generated in this study will allow clinicians to make accurate predictions of LR and RR for NPC treated with radical IMRT. Additional studies are required to determine whether it can be applied to other patient groups.

\section{Grant Support}

This work was supported by grants from the Health \& Medical Collaborative Innovation Project of Guangzhou City, China (No. 201400000001), the National Science \& Technology Pillar Program during the Twelfth Five-year Plan Period (No. 2014BAI09B10), the National Natural Science Foundation of China (No. 81402532, 81372409), and the Science and Technology Project of Guangzhou City, China (No. 132000507). The funding source(s) had no involvement in study design; collection, analysis and interpretation of data; writing of the report; or decision to submit the article for publication.

\section{Competing Interests}

The authors have declared that no competing interest exists.

\section{References}

1. Jemal A, Bray F, Center MM, et al. Global cancer statistics. CA Cancer J Clin. 2011;61(2):69-90.

2. Haberer-Guillerm S, Touboul E, Huguet F. Intensity modulated radiation therapy in nasopharyngeal carcinoma. European annals of otorhinolaryngology, head and neck diseases. 2015;132(3):147-51.

3. Chua MLK, Wee JTS, Hui EP, et al. Nasopharyngeal carcinoma. The Lancet. 2016;387(10022):1012-24

4. Xu T, Tang J, Gu M, et al. Recurrent nasopharyngeal carcinoma: a clinical dilemma and challenge. Current oncology. 2013;20(5): e406-19.

5. Chua DT, Sham JS, Leung LH, et al. Re-irradiation of nasopharyngeal carcinoma with intensity-modulated radiotherapy. Radiotherapy and oncology: journal of the European Society for Therapeutic Radiology and Oncology. 2005;77(3):290-4.

6. Zheng XK, Ma J, Chen LH, et al. Dosimetric and clinical results of three-dimensional conformal radiotherapy for locally recurrent nasopharyngeal carcinoma. Radiotherapy and oncology: journal of the European Society for Therapeutic Radiology and Oncology. 2005;75(2):197-203.

7. Li JC, Hu CS, Jiang GL, et al. Dose Escalation of Three-dimensional Conformal Radiotherapy for Locally Recurrent Nasopharyngeal Carcinoma: A Prospective Randomised Study. Clin Oncol (R Coll Radiol). 2006 May;18(4):293-9.

8. Qiu S, Lin S, Tham IWK, et al. Intensity-Modulated Radiation Therapy in the Salvage of Locally Recurrent Nasopharyngeal Carcinoma. Int. J. Radiation Oncology Biol. Phys. 2012;83(2):676-83. 
9. Chua DT, Wu SX, Lee V, et al. Comparison of single versus fractionated dose of stereotactic radiotherapy for salvaging local failures of nasopharyngeal carcinoma: a matched-cohort analysis. Head \& neck oncology. 2009;1:13.

10. Seo Y, Yoo H, Yoo S, et al. Robotic system-based fractionated stereotactic radiotherapy in locally recurrent nasopharyngeal carcinoma. Radiotherapy and oncology: journal of the European Society for Therapeutic Radiology and Oncology. 2009;93(3):570-4.

11. Roeder F, Zwicker F, Saleh-Ebrahimi L, et al. Intensity modulated or fractionated stereotactic reirradiation in patients with recurrent nasopharyngeal cancer. Radiat Oncol. 2011;6:22.

12. Lin JCh, Wang WY, Chen KY, et al. Quantification of Plasma Epstein-Barr Virus DNA in Patients with Advanced Nasopharyngeal Carcinoma. N Engl J Med 2004;350:2461-70.

13. Ng WT, Lee MC, Chang AT, et al. The impact of dosimetric inadequacy on treatment outcome of nasopharyngeal carcinoma with IMRT. Oral oncology. 2014;50(5):506-12.

14. Li JX, Huang SM, Jiang $\mathrm{XH}$, et al. Local failure patterns for patients with nasopharyngeal carcinoma after intensity-modulated radiotherapy. Radiat Oncol. 2014;9:87

15. Wan $\mathrm{XB}$, Wei $\mathrm{L}, \mathrm{Li} \mathrm{H}$, et al. High pretreatment serum lactate dehydrogenase level correlates with disease relapse and predicts an inferior outcome in locally advanced nasopharyngeal carcinoma. European journal of cancer. 2013;49(10):2356-64.

16. Cheng SH, Tsai SY, Horng CF, et al. A prognostic scoring system for locoregional control in nasopharyngeal carcinoma following conformal radiotherapy. Int. J. Radiation Oncology Biol. Phys. 2006;66(4):992-1003.

17. Xia $\mathrm{P}, \mathrm{Fu} \mathrm{KK}$, Wong GW, et al. Comparison of treatment plans involving intensity modulated radiotherapy for nasopharyngeal carcinoma. Int. J. Radiation Oncology Biol. Phys. 2000;48(2):329-337.

18. Tham IW, Hee SW, Yeo RM, et al. Treatment of nasopharyngeal carcinoma using intensity-modulated radiotherapy-the national cancer centre singapore experience. Int. J. Radiation Oncology Biol. Phys. 2009;75(5):1481-6.

19. Chen L, Mao YP, Xie FY, et al. The seventh edition of the UICC/AJCC staging system for nasopharyngeal carcinoma is prognostically useful for patients treated with intensity-modulated radiotherapy from an endemic area in China. Radiotherapy and oncology: journal of the European Society for Therapeutic Radiology and Oncology. 2012;104(3):331-7.

20. Kuang WL, Zhou Q, Shen LF. Outcomes and prognostic factors of conformal radiotherapy versus intensity-modulated radiotherapy for nasopharyngeal carcinoma. Clinical \& translational oncology: official publication of the Federation of Spanish Oncology Societies and of the National Cancer Institute of Mexico. 2012;14(10):783-90.

21. Tao CJ, Liu X, Tang LL, et al. Prognostic scoring system for locoregional control among the patients with nasopharyngeal carcinoma treated by intensity-modulated radiotherapy. Chin J Cancer. 2013;32(9):494-501.

22. Balachandran VP, Gonen M, Smith JJ, et al. Nomograms in oncology: more than meets the eye. The Lancet Oncology. 2015;16(4):e173-e80.

23. Iasonos A, Schrag D, Raj GV, et al. How to build and interpret a nomogram for cancer prognosis. Journal of clinical oncology: official journal of the American Society of Clinical Oncology. 2008;26(8):1364-70.

24. Bendifallah $S$, Ballester M, Uzan C, et al. Nomogram to predict recurrence in patients with early- and advanced-stage mucinous and serous borderline ovarian tumors. American journal of obstetrics and gynecology. 2014;211(6):637-6.

25. $\mathrm{Li}$ WF, Sun $Y$, Chen $M$, et al. Locoregional extension patterns of nasopharyngeal carcinoma and suggestions for clinical target volume delineation. Chinese journal of cancer. 2012;31(12):579.

26. Li JX, Lu TX, Huang Y, et al. Clinical features of 337 patients with recurrent nasopharyngeal carcinoma. Chinese Journal of Cancer. 2010;29(1):76-80.

27. Cho JK, Lee GJ, Yi KI, et al. Development and external validation of nomograms predictive of response to radiation therapy and overall survival in nasopharyngeal cancer patients. European journal of cancer. 2015;51(10):1303-11.

28. Kong F, Ying H, Du C, et al. Patterns of local-regional failure after primary intensity modulated radiotherapy for nasopharyngeal carcinoma. Radiat Oncol. 2014;9:60.

29. B Hong, VWY Lui, M Hashiguchi, et al. Targeting tumor hypoxia in nasopharyngeal carcinoma. Head \& Neck. 2013;35(1):133-145.

30. Kuang DM, Zhao Q, Wu Y, et al. Peritumoral neutrophils link inflammatory response to disease progression by fostering angiogenesis in hepatocellular carcinoma. Journal of hepatology. 2011;54(5):948-55.

31. He JR, Shen GP, Ren ZF, et al. Pretreatment levels of peripheral neutrophils and lymphocytes as independent prognostic factors in patients with nasopharyngeal carcinoma. Heas \& Neck. 2012;34(12):1769-1776.

32. Wang QQ. Tumor-associated neutrophils in tumor development and progression. Chinese Journal of Cancer Biotherapy. 2010;17(5):487-491.

33. Lee N, Harris J, Garden AS, et al. Intensity-modulated radiation therapy with or without chemotherapy for nasopharyngeal carcinoma: radiation therapy oncology group phase II trial 0225. Journal of Clinical Oncology. 2009;27(22):3684-90.

34. Edge, S. B. et al. AJCC Cancer Staging Manual. (Springer, New York, 2010). 